\title{
Le Wallstein de Benjamin Constant, entre dramaturgie des Lumières et Romantisme
}

\section{Pierre Frantz}

\section{(2) OpenEdition}

Journals

\section{Édition électronique}

URL : http://journals.openedition.org/rief/240

DOI : $10.4000 /$ rief.240

ISSN : 2240-7456

\section{Éditeur}

Seminario di filologia francese

\section{Référence électronique}

Pierre Frantz, "Le Wallstein de Benjamin Constant, entre dramaturgie des Lumières et Romantisme », Revue italienne d'études françaises [En ligne], 3 | 2013, mis en ligne le 15 décembre 2013, consulté le 19 avril 2019. URL : http://journals.openedition.org/rief/240 ; DOI : 10.4000/rief.240

Ce document a été généré automatiquement le 19 avril 2019

\section{(c) $($ i) $(9)$}

Les contenus de la RIEF sont mis à disposition selon les termes de la Licence Creative Commons Attribution - Pas d'Utilisation Commerciale - Pas de Modification 4.0 International. 


\title{
Le Wallstein de Benjamin Constant, entre dramaturgie des Lumières et Romantisme
}

\author{
Pierre Frantz
}

1 La comparaison entre le théâtre français du tournant du siècle et celui de l'Allemagne, on l'a souvent rappelé, est plutôt cruelle pour le premier. D'un côté Schiller et Goethe, de l'autre Lemercier, Chénier ou Luce de Lancival. Avec Madame de Staël, Schlegel et Benjamin Constant, le sens des transferts culturels s'est durablement inversé et la présence des dramaturges allemands, insistante depuis les années 1780, depuis Friedel, Juncker et Liebaut, possède dès lors un rayonnement remarquable. Les échanges s'étaient intensifiés dans le dernier tiers du XVIII siècle. Un exemple : pour son Don Carlos, Schiller part, comme Louis-Sébastien Mercier, de la nouvelle de Saint-Réal et dialogue indirectement avec Mercier dont il traduit partiellement le Portrait de Philippe II. En sens inverse, ses Räuber sont adaptés en France par La Martellière qui en tire deux pièces ; enfin sa trilogie de Wallenstein est commentée par Benjamin Constant en 1809 dans la préface de la curieuse tragédie qu'il en a tirée, puis par Madame de Staël dans De l'Allemagne. Benjamin Constant revient sur Wallenstein et sur son propre Wallstein un an avant sa mort en 1829 : il réédite dans les Mélanges de littérature et de politique sa préface de Wallstein, amendée de façon significative, et publie un article de Réflexions sur la tragédie dans le tome VII de la Revue de Paris. Cette publication avait suscité des réactions diverses et contrastées. On distinguera deux commentaires intéressants, l'un de Madame de Staël, l'autre de Prosper de Barante, qui avait lui-même donné une traduction de Schiller, et pas moins de cinq articles de Guizot (repris après sa mort dans Le temps passé en 1887). Sans doute les représentations en 1828 d'une tragédie de Pierre Chaumont de Liadières, sous le même titre avaient-elles dû inspirer à Constant ce retour sur son œuvre dramatique. Celle-ci, peu prisée par la critique, avait connu pourtant, de façon surprenante, un réel succès de librairie. Car Constant n'avait pas voulu - pas osé peut-être - donner sa pièce aux comédiens en 1809 et l'avait réservée à la lecture. Il avait pu lui conserver ainsi une liberté expérimentale, affranchie des contraintes, qu'un passage par la Comédie-Française 
eussent fait peser sur elle, même si les variantes recensées témoignent que le souci de la représentation était bien présent à son esprit.

Les réflexions de Constant frappent par leur grande portée théorique et par la lucidité de leur auteur. Wallstein a-t-il échoué - comme le suggèrent Benjamin Constant et Germaine de Staël - parce que son auteur avait voulu soumettre sa pièce au lit de Procuste de la dramaturgie néoclassique, celle de Voltaire et celle des Lumières? Cette idée semble aller de soi ${ }^{1}$. Nous tenterons pourtant moins d'écarter ce jugement critique que d'en proposer une interprétation plus nuancée. La première raison en est que les propositions dramaturgiques du XVIII ${ }^{e}$ siècle, celles qui sont formulées par les philosophes des Lumières, Diderot, Voltaire ou Beaumarchais, et qu'on serait tenté d'incriminer, sont diverses et grosses de plusieurs avenirs différents. C'est le combat entre les classiques et les romantiques qui a caricaturé la représentation qu'on s'est faite du théâtre des Lumières. Pour ce faire, je procéderai à plusieurs confrontations, entre Wallstein et son modèle allemand, d'un côté et sa (ou ses) relecture(s) française(s) de l'autre.

La première difficulté rencontrée par Constant est celle de la langue et du vers alexandrin tragique dont le modèle lui a été légué par le XVIII ${ }^{e}$ siècle encore plus que par le XVII ${ }^{e}$. C'est là un fait trop connu pour que l'on s'y attarde. Comme l'explique Madame de Staël, les Français veulent faire entendre des beautés non seulement dans chaque scène mais à chaque vers. D'où l'abus fatigant de périphrases, d'inversions et de formules pompeuses. Constant évite d'écrire, par exemple, "la France». Le rude général s'exprime ainsi lorsqu'il évoque ses soldats : "Quelques-uns dans la Gaule ont reçu la naissance » ${ }^{2}$. Et si l'on va jusqu'à dire "l'Allemagne ", ses guerriers sont nommés des "Germains " ${ }^{3}$. Les malsonnantes trompettes, c'est " l'airain qui retentit » ${ }^{4}$, mais, plus loin, l'airain ce sont les canons :

Et cent bouches d'airain sur ses pâles soldats

Du haut de votre camp vomissent le trépas. ${ }^{5}$

4 Plutôt que de choisir un « couvent » pour retraite, Thecla, l'héroïne, évoque ces « lieux retirés, par la religion aux larmes consacrés ». Constant l'affirme encore en 1809, «La pompe inséparable des alexandrins nécessite dans l'expression une noblesse soutenue $»^{6}$. On a reproché pourtant à Constant d'être parfois familier : il ne l'a pas été assez souvent.

Constant s'est montré le plus novateur dans la conception de l'action de sa tragédie. En 1809 il admirait et s'étonnait de la façon dont Schiller avait conçu l'action théâtrale. Il y avait là, sans aucun doute de quoi surprendre les Français. Pourtant quelque chose était dans l'air à la fin de l'Ancien Régime. Beaumarchais avait conçu une trilogie. Le souvenir des trilogies antiques lui était venu à l'esprit, comme on le voit dans la "Lettre modérée » qui précède Le Barbier de Séville. C'est à la fin de l'écriture de cette comédie qu'il conçoit la suite et la déploie sur la toile de fond de ce que Martine de Rougemont a appelé «le roman » de la famille Almaviva. De même l'adaptation des Räuber par La Martelière se développe sur deux pièces, Robert, chef des brigands et Le Tribunal redoutable. Picard fait jouer au Théâtre de la Nation en 1791 Le Passé, le présent et l'avenir, une comédie ou plus exactement une trilogie, composée de trois pièces en un acte ${ }^{7}$.

Le Passé, le présent et l'avenir ne ressemblent donc à aucune de mes autres productions par la création, le plan, les caractères et même le style. Dans cet ouvrage, je prétendais ne m'astreindre à aucune règle, et je bravai singulièrement la poétique d'Aristote pour ce qui regarde les unités de temps, de lieu et d'action. Lorsque je me rappelle la date de cette comédie, qui fut reçue au Théâtre-Français le 30 juillet 1791, je suis presque fier d'avoir hasardé une innovation que d'autres 
s'attribuent par brevet d'invention aujourd'hui. Il est vrai que ma comédie favorite, [...] est à peu près oubliée depuis trente sept ans ; mais elle ne mérite pas cet oubli et mes amis qui la connaissent partagent la bonne opinion que j'en ai. ${ }^{8}$ précisément en 1828, l'année de sa mort, Les Éphémères, "tragi-comédie en 3 actes et en prose, précédée d'un prologue et suivie d'un épilogue ${ }^{9}$. La trilogie de Schiller, Benjamin Constant le remarque, comporte un prologue sans action, Le Camp de Wallenstein, une action interrompue, Les Piccolomini, et un dénouement, La Mort de Wallenstein, qui, à lui seul, occupe la plus grande partie du drame. Il n'y a ni unité de temps ni de lieu : si les deux premières pièces et les trois premiers actes de la dernière se déroulent devant ou à Pilsen, les deux derniers actes se jouent à Eger. Mais en vérité la question n'était pas seulement celle de l'étendue de l'action ou celle des règles : c'est la nature même de l'action dramatique qui change avec l'abandon du système des règles et l'irruption d'une temporalité nouvelle.

Réflexions sur la tragédie de 1829. À cet égard, ce dernier texte est celui qui va le plus loin de toute la littérature romantique. Il esquisse certaines idées que l'on rencontrera chez Antoine, Craig et Zola. Les concepts poétiques auxquels on a recours dans l'aristotélisme français pour décrire l'action, « exposition, nœud, péripétie, catastrophe, dénouement » prennent appui sur une représentation linéaire, celle du discours ou celle qu'exprime parfaitement l'image des fils qui forment le nœud. Ils impliquent la formulation d'une dynamique horizontale, celle des fils, celle d'une arme qui frappe, peripeteia, ou verticale catastrophè, mais toujours linéaire et continue. Dans cette perspective, il n'y a guère de différence entre l'intrigue et l'action. Elles se distinguent cependant, principalement en fonction des différences dans la conscience, ou dans le savoir, qu'ont les personnages ou les spectateurs en ce qui concernent les événements auxquels ils assistent. C'est pourquoi les quiproquos et les coups de théâtre tiennent dans les drames et la dramaturgie classique une place si importante. C'est au point qu'on tend à identifier le «dramatique » avec ce type de fonctionnement, comme on le voit chez des théoriciens comme Brecht, Szondi ou Lehmann. Dès le milieu du XVIII ${ }^{\mathrm{e}}$ siècle ces formulations théoriques entrent en concurrence avec d'autres présentations, pour l'essentiel liées à la conception des tableaux et des personnages. Benjamin Constant se réfère à Diderot dans son texte le plus abouti, ses Réflexions sur la tragédie de 1829. Il commence son article par un rappel du Discours sur la poésie dramatique et cite le passage dans lequel Diderot propose de substituer aux «caractères » les «conditions ». Le texte est assez célèbre pour ne pas le citer ici. Mais, on ne l'a pas noté très souvent, l'auteur du Fils naturel étend la réflexion sur les personnages à la question de l'action et en tire une conception dont vont s'emparer Madame De Staël (sans le dire) et Benjamin Constant, qui reconnaît sa dette diderotienne :

C'est du caractère qu'on tirait toute l'intrigue; on cherchait en général les circonstances qui le faisaient ressortir, et l'on enchaînait ces circonstances. C'est sa condition, ses devoirs, ses avantages, ses embarras, qui devraient servir de base à l'ouvrage. ${ }^{10}$

8 Si Constant souligne les insuffisances et les petitesses de la théorie du drame, il en perçoit la portée :

Sous la pensée de Diderot, j'en vois une plus vaste, et je veux essayer de la développer. [...] Trois choses peuvent servir de base aux compositions tragiques, la peinture des passions, le développement des caractères, et l'action de la société, 
telle qu'à chaque époque elle est constituée, et telle qu'elle agit sur le caractère et les passions. ${ }^{11}$

Le Wallenstein de Schiller, son Guillaume Tell, l'Egmont et le Torquato Tasso lui paraissent relever d'un genre nouveau de tragédies, celui qui déduit l'action du caractère. La variété des exemples qu'il cite alors, rapprochant ces drames du Mahomet de Voltaire et même de Britannicus, montre toute l'extension qu'il donne à sa théorie. Du troisième genre évoqué, qui résulte de l'action de la société, il avoue n'avoir aucun exemple, mais il tire principalement des réussites allemandes dans le second genre, l'idée d'un type de drame, qui adopterait pour principe recteur l'action de la société sur les caractères et les passions.

C'est l'ensemble qu'il faut retracer, en même temps et aussi fortement que la figure isolée qui doit dominer sur le premier plan. ${ }^{12}$

10 Mercier, dès 1773, disait de même, usant de la métaphore picturale, que le temps était venu de substituer le «tableau » au « portrait $»^{13}$. Il dénonçait aussi, selon une métaphore politique significative, une forme tragique dans laquelle le caractère agissait sur l'action comme un despote devant qui les autres caractères tremblaient ${ }^{14}$. Madame de Staël aperçoit bien, elle aussi, dans son évocation du Wallensteins Lager, un autre univers d'action dramatique.

Le camp de Walstein, qui est la première des trois représente les effets de la guerre sur la masse du peuple et de l'armée, la seconde (Les Piccolomini) montre les causes politiques qui préparent les dissensions entre les chefs; et la troisième, la Catastrophe est le résultat de l'enthousiasme et de l'envie que la réputation de Walstein avait excités. ${ }^{15}$

11 Le concept d'action se dégage de celle du caractère ou, si on préfère, du personnage. Pour parler le langage des sémiologues, l'actant ne saurait plus se confondre avec un personnage, alors que, dans le système classique, les deux notions, sans se confondre, se recouvrent l'une l'autre le plus souvent. L'action ne se représente plus selon une ligne ou un fil qu'on noue, mais en volume. Elle se déduit d'un montage. Les scènes du Camp de Wallenstein ou celles des Piccolomini pourraient être placées dans un ordre différent. Et Diderot proposait déjà de s'affranchir de la liaison des scènes. Chez les Stürmer und Dränger et chez les romantiques allemands, il en va ainsi : Die Sodaten de Lenz ou Woyzeck de Büchner présentent des séquences de scènes séparées. Montage encore si l'on pense aux « trilogies » évoquées tout à l'heure. Si l'on prend au sérieux le « roman de la famille Almaviva ", celui-ci existe à l'arrière-plan des trois pièces qui le composent, même si, très habilement, Beaumarchais a, en quelque sorte, superposé une conception nouvelle et une approche traditionnelle.

12 Mais ce que Benjamin Constant voit nettement en 1829, l'avait-il entièrement compris vingt ans plus tôt alors qu'il écrivait son Walstein? Oui en un sens, car il observe justement, commentant Schiller :

Les scènes se suivent sans que rien les enchaîne l'une à l'autre: mais cette incohérence est naturelle ; c'est un tableau mouvant où il n'y a ni passé, ni avenir. Cependant le génie de Walstein préside à ce désordre apparent. ${ }^{16}$

13 Mais il s'empresse de rattacher l'action au caractère de Walstein, alors que ce dernier, il ne s'en avise pas alors, est au moins autant un produit des camps et de la société militaire dont il est issu qu'un héros qui rayonne sur cette société et l'entraîne à sa suite. Du reste, c'est précisément là sa faiblesse. Walstein reste hésitant et est, in fine, le jouet de la cour et des officiers. Si le théâtre montre une "action", au sens du verbe grec drân, Wallenstein n'en est pas le sujet. Mais précisément c'est l'action même qui est au cœur du 
questionnement ouvert par le drame. Rien de plus théâtral. Rien de plus étymologiquement dramatique. Schiller est ici proche de Sophocle. L'action se retourne et se questionne donc: «qu'est-ce qu'une action?». À certains égards, Lorenzaccio est déjà en puissance dans Wallenstein de Schiller. Mais en est-il de même dans Wallstein?

Benjamin Constant, en 1809, avait pensé que les Français ne pouvaient encore accepter un tel système dramatique germanique. Il aurait pu aller jusqu'au bout de la démarche romantique expérimentale, et adapter Schiller en restant proche de son modèle, puisqu'il se doutait bien qu'il ne pourrait faire jouer sa pièce. Il aurait pu à l'inverse, comme Pierre Chaumont de Liadières, reprendre le sujet en en faisant une tragédie classique. Il est resté au milieu du gué. Sa tragédie se déroule en une seule journée, le 25 février 1634, dans la dix-huitième année de la guerre de Trente Ans. Du Camp de Wallenstein il ne retient que quelques discussions d'officiers avec l'envoyé de la cour et n'évoque la vie militaire et l'amour des soldats pour leur chef que par quelques allusions, au détour d'un vers ou d'une tirade. Liadières, quant à lui, se contente d'utiliser le pittoresque du décor, évoqué dans une copieuse didascalie initiale, pour évoquer le camp. Pratiquement, Constant commence donc sa pièce avec les Piccolomini. L'intrigue politique prend le premier pas. L'auteur n'a pourtant pas renoncé à l'histoire d'amour de Thekla et Max Piccolomini (rebaptisé Alfred) ; mais cette histoire d'amour n'est pas traitée comme une passion à la française. Elle ne commande rien dans l'action. La défection d'Alfred et l'approbation de son amante répondent bien à celle de Max Piccolomini et de l'héroïne de Schiller. Benjamin Constant remarque donc que, dans la dramaturgie allemande, l'amour n'est pas une passion mais un sentiment quasi religieux, qui confère au tout une couleur poétique. L'intrigue politique et l'intrigue amoureuse ne forment pas vraiment un nœud: Alfred, pas plus que Max, ne joue un rôle déterminant contre Wallstein. Ses désarrois intimes restent de nature morale. Ici, Constant a repris quelques éléments de dramaturgie cornélienne pour trouver une sorte d'équivalent à la pièce originelle. Il a renoncé aussi à divers éléments de type shakespearien, présents dans le drame de Schiller. Il confesse avoir renoncé ainsi au dialogue pittoresque des deux soldats assassins. Impossible de faire autrement: en 1809, il n'était réellement pas possible de placer un dialogue vulgaire entre deux soudards au milieu d'une tragédie :

[...] le langage de ces assassins est vulgaire, comme leur état et leurs sentiments.

Leur prêter des expressions relevées, c'eût été manquer à la vérité des caractères et

dans ce cas la noblesse du dialogue serait devenue une inconvenance. ${ }^{17}$

15 Tous les personnages populaires de Schiller, qui donnent vraiment à l'action sa signification, disparaissent. Les compagnons du héros, dont Schiller a souligné les origines diverses, dont il met en avant la sincérité mais aussi la fragilité de leur adhésion à la figure du chef, sont des officiers. Ce sont des sujets qui, chez Constant, mènent l'action, alors que chez Schiller ils sont dispersés en une force aveugle et anonyme, destinée, société, histoire peu importe.

On accordera une importance particulière à un événement qui est traité différemment chez chacun des trois dramaturges. Chez Schiller, la trahison de Wallstein est attestée par les papiers secrets de Terzky, saisis sur un envoyé secret :

[...] Qui a-t-on livré?

TERZKY

Celui qui sait notre secret, celui qui sait ce que nous avons négocié avec les Saxons, les Suédois, entre les mains de qui tout est passé. ${ }^{18}$ 
17 Wallstein ne peut que maudire à plusieurs reprises le «fâcheux hasard » qui l'oblige à agir :

Quoi que je fasse, je suis et je demeure

Pour eux un traitre à son pays.

Et même si je retourne en toute loyauté

À mon devoir, il est trop tard.

ILLO

Ceci te perdra. On ne l'attribuera point

À ta loyauté, mais à ton impuissance. ${ }^{19}$

Et, dans un monologue, Wallstein se retourne sur sa vie :

Derrière moi la route a disparu,

Les œuvres dressent un mur

Une Tour qui me barre le chemin du retour [...]

Le double sens

De la vie m'accuse et le soupçon,

Le perfide interprète irait jusqu'à noircir

L'acte pur né d'une source claire. ${ }^{20}$

À l'évidence, il y a là le sens même de l'action du drame.

Constant recule d'un cran par rapport à Schiller. Le comte Terzky, intime ami de Wallstein a pris les devants dans la trahison, alors que Wallstein hésitait encore :

Hélas! vous blâmerez mon ardeur imprudente,

Seigneur, je le prévois. De vos ordres chargé

Avec le Suédois, je m'étais engagé.

Vous même vous le saviez; mais votre incertitude

Semblait, de l'oublier, s'être fait une étude. ${ }^{21}$

Le dernier pas, en quelque sorte, il ne l'a pas fait lui-même. L'auteur suit Schiller en présentant ce curieux héros dépossédé de son acte mais il ne donne pas à cette dépossession la signification profonde et mystérieuse de la pièce allemande. Sans doute celle-ci s'en trouve-t-elle en quelque sorte laïcisée mais, on le voit, Constant veut que son héros paraisse moins coupable. Il désindividualise en quelque sorte pourtant l'action. Le traître Butler a lui aussi agi en allant plus loin que les instructions que lui avaient données Gallas et en faisant tuer Wallstein. "Qu'ai-je fait après tout, qu'achever votre ouvrage ? $»^{22}$ lui dit-il. La répétition, la symétrie Gallas / Wallstein, thématise en quelque sorte la motivation de l'action. Les choses ne vont pas où l'on croit. Les mots dépassent les pensées et les actes sont plus parlants que les dialogues. 
Liadières, la surprise de l'espion ne change pas grand chose. Wallstein a pris sa décision lorsqu'il a appris les injures dont sa femme et sa fille ont été l'objet à la cour de Vienne. C'est donc une action emportée par la passion: tout devient une affaire de vengeance, d'ambition, dans laquelle l'épouse de Wallstein (absente de la pièce de Benjamin Constant) joue un rôle important. Liadières s'emploie à escamoter la trahison du héros. Celle même de Butler, qui livre son ami par ambition déçue, est curieusement traitée. À la fin, Butler, ému par la sincérité du vieux général, part pour stopper les assassins qu'il avait engagés, mais il est lui-même tué par Albert, l'équivalent du Alfred de Constant et du Max de Schiller, qui ignore tout de son revirement. Les assassins feront leur œuvre. Albert n'est pas tué : il épouse Thekla et s'occupe de sa belle mère... Dans un avant-propos, Liadières se targue d'avoir su éviter d'écrire une "tragédie biographique » comme celles de ses prédécesseurs. Il est impossible, dit-il, de composer une tragédie digne de ce nom sur leur modèle.

Wallstein peint tout entier, ambitieux, jaloux, inquiet, superstitieux, décidé dans ses paroles, incertain dans ses actions, incapable de prendre un parti lorsqu'il a tout préparé pour le succès, serait-il un personnage dramatique? Telles sont les questions qu'il ne m'appartient pas de résoudre. De plus habiles que moi l'ont essayé vainement et l'essaieront vainement encore. On ne nous a opposé jusqu'ici que de brillantes théories. Tant qu'un chef-d'œuvre ne les aura pas justifiés [sic], qu'on nous permette de préférer la poétique de Racine à celle de Shakespeare. ${ }^{23}$

Bref, avec la tragédie de Liadières, on revient à une conception "aristotélicienne " de l'action, avec coups de théâtre, péripéties, catastrophe. C'est une affaire de cour qui tourne mal. Les critiques classiques ont reproché à Constant d'avoir conservé, quoique de façon atténuée, la dimension superstitieuse du héros. Quelques vers font allusion en effet, de façon récurrente, à la fatalité et aux astres.

Instinct confus, faut-il encore te prendre pour guide?

Es-tu la voix du ciel ? Non le ciel est perfide.

L'amitié m'a trahi. Les astres m'ont trompé.

D'une éternelle nuit l'homme est enveloppé.

On interroge en vain l'inflexible nature.

Il faut donc marcher seul dans cette route obscure ${ }^{24}$

Son instinct poétique l'avertissait évidemment que Schiller avait eu raison de donner ce caractère à son héros. La force qui agit et que Constant tentera d'identifier à l'action de la société, est plus obscure en 1809. Impossible non plus de la prendre pour la Providence. Wallstein la nomme destin :

Impérieux destin, ton ordre est satisfait !

Tu m'entrainais au Trône et j'y monte en effet.

Mais je sens dans mon cœur se flétrir l'espérance.

Je ne t'invoque plus. Je cède à ta puissance.

Comme un poids étranger je reçois tes bienfaits. ${ }^{25}$ 

s'étale sur un demi siècle que de la fascination exercée par les Allemands sur un groupe d'écrivains longtemps placé dans une situation d'intermédiaires. La force poétique de Schiller a exercé une attraction puissante qui a infléchi une évolution, dont témoignait déjà la trilogie de Beaumarchais ou le Pinto de Louis Népomucène Lemercier. Si Benjamin Constant ne s'y est pas abandonné complètement alors qu'il en avait une intelligence très fine, c'est sans doute à cause des résistances du public français, qu'il ne se sentait pas la force de défier, même à la seule lecture. Comme, après lui, Ludovic Vitet ou Musset, le théâtre dans un fauteuil lui a permis d'ouvrir un chantier dramaturgique que les romantiques français, après lui, n'ont pas achevé non plus, celui du drame historique. Les formules trouvées par Victor Hugo n'appartiennent pas en effet à une esthétique comme celle de Schiller. Seul s'en est approché Musset, encore a-t-il fallu attendre 1896, bien après la fin du romantisme pour que Lorenzaccio trouve sur la scène son achèvement.

\section{NOTES}

1. Sur ces réactions, voir Wallstein, tragédie en cinq actes et en vers de Benjamin Constant, édition critique par J.-R. Derré, Paris, Les Belles Lettres, «Bibliothèque de la Faculté des Lettres de Lyon », 1965, p. 42, notamment.

2. Ibid., p. 129.

3. Ibid., p. 130.

4. Ibid., p. 79.

5. Ibid., p. 88.

6. B. Constant, Quelques Réflexions sur la tragédie, dans Wallstein, op. cit., p. 52.

7. L.-B. Picard, Le Passé, Le Présent, L'Avenir, Théâtre de la Nation, 1791.

8. Id., Théâtre Républicain, posthume et inédit, Paris, Madame Charles-Béchet, J.-N. Barba, Lecointe et Pougin, 1832, p. 22. 
9. Les Éphémères, «tragi-comédie en 3 actes et en prose, précédée d'un prologue et suivie d'un épilogue », par M. Picard,... et M. [Mazères]... [Paris, Odéon, 14 février 1828].

10. B. Constant, Réflexions sur la tragédie, dans CEuvres, texte présenté et annoté par Alfred Roulin, Paris, Gallimard, « Bibliothèque de la Pléiade », 1957, p. 935.

11. Ibid., p. 936.

12. Ibid., p. 947.

13. L. S. Mercier, Du Théâtre [1773], édition annotée par P. Frantz, dans Mon Bonnet de nuit, Du Théâtre, dir. J.-C. Bonnet, Paris, Mercure de France, 1999, p. 1203-1204.

14. L. S. Mercier, Du Théâtre, op. cit., p. 1171.

15. Madame de Staël, De l'Allemagne, t. I, Paris, Flammarion, « GF », 1968, p. 277.

16. B. Constant, «Quelques réflexions sur la tragédie de Walstein », dans Walstein, op. cit., p. 50.

17. Ibid., p. 58.

18. F. Schiller, Wallenstein, trad. G. Darras, Paris, L'Arche, 2005, p. 167.

19. Ibid., p. 169.

20. Ibid., p. 171.

21. B. Constant, Wallstein, op. cit., II, 2, p. 99.

22. Ibid., p. 185.

23. P.-Ch. Liadières, Wallstein, Paris 1829, [avant-propos].

24. B. Constant, Wallstein, op. cit., V, 10, p. 181.

25. Ibidem.

26. Benjamin Constant, Réflexions sur la Tragédie (1829), cit., p. 945.

INDEX

Mots-clés : Lumières, romantisme, Schiller, action dramatique, théorie dramatique 